\title{
An Exhaustive Approach to the Coupling Matrix Synthesis Problem Application to the Design of High Degree Asymmetric Filters
}

\author{
Richard J. Cameron ${ }^{(1)}$, Jean-Charles Faugere ${ }^{(2)}$, Fabrice Rouillier ${ }^{(3)}$ and Fabien Seyfert ${ }^{(3)}$ \\ ${ }^{(1)}$ Com Dev Space (UK), ${ }^{(2)}$ Univ. Paris VI (France), ${ }^{(3)}$ INRIA (France)
}

\begin{abstract}
In this paper a new approach to the synthesis of coupling matrices for microwave filters is presented. The new approach represents an advance on existing direct and optimization methods for coupling matrix synthesis in that it will exhaustively discover all possible coupling matrix solutions for a network if more than one exists. This enables a selection to be made of the set of coupling values, resonator frequency offsets, parasitic coupling tolerance etc that will be best suited to the technology it is intended to realize the microwave filter with. To demonstrate the use of the method, the case of the recently introduced 'extended box' coupling matrix configuration is taken. The extended box is a new class of filter configuration adapted to the synthesis of asymmetric filtering characteristics of any degree. For this configuration the number of solutions to the coupling matrix synthesis problem appears to be high and offers therefore some flexibility that can be used during the design phase. We illustrate this by carrying out the synthesis process of two asymmetric filters of $8^{\text {th }}$ and $10^{\text {th }}$ degree. In the first example a ranking criterion is defined in anticipation of a dual mode realization and allows the selection of a "best" coupling matrix out of 16 possible ones. For the $10^{\text {th }}$ degree filter a new technique of approximate synthesis is presented yielding some simplifications of the practical realization of the filter as well as of its computer aided tuning phase.
\end{abstract}

Index Terms - Coupling matrix, filter synthesis, bandpass filter, Groebner basis, inverted characteristic, multiple solutions.

\section{INTRODUCTION}

In reference [1], a synthesis method for the 'Box Section' configuration for microwave filters was introduced. Box sections are able to realize a single transmission zero each, and have an important advantage that no 'diagonal' inter-resonator couplings are required to realize the asymmetric zero, as would the equivalent trisection. Also the frequency characteristics are reversible by retuning the resonators alone, retaining the same values and topology of the inter-resonator couplings.

The first feature leads to particularly simple coupling topologies, and is suitable for realization in the very compact waveguide or dielectric dual-mode resonator cavity, whilst the ability to reverse the characteristics by retuning makes the box-filter useful for diplexer applications, the same structure being usable for the complementary characteristics of the two channel filters.

Reference [1] continued on to introduce the extended box configuration for filter degrees $N>4$, able to realize a maximum of $(N-2) / 2(N$ even) or $(N-3) / 2$ ( $N$ odd) symmetric or asymmetric transmission zeros. Fig. 1 gives extended box networks of even degree 4 (basic box section), 6, 8 and 10, showing the particularly simple ladder network form of the extended box configuration. In each case, the input and output are from opposite corners of the ladder network. The extended box network also retains the property of giving lateral inversion of the frequency characteristics by retuning of the resonators alone.

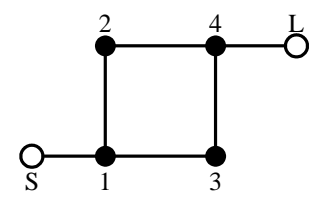

(a)

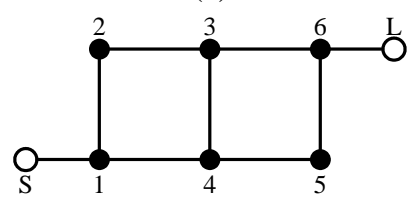

(b)

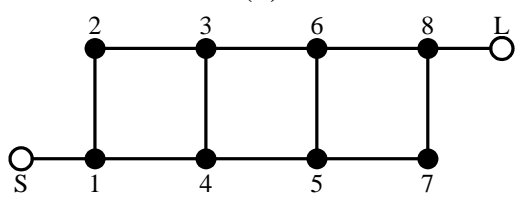

(c)

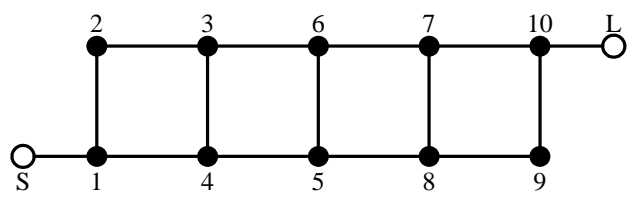

(d)

Fig. 1. Coupling and routing diagrams for extended box section networks: (a) $4^{\text {th }}$ degree (basic box section) (b) $6^{\text {th }}$ degree (c) $8^{\text {th }}$ degree (d) $10^{\text {th }}$ degree.

The prototype coupling matrix for the extended box network may be easily synthesized in the folded or 'arrow' forms. However it appears that there is no simple closed form equation or procedure that may be used to transform the folded or arrow coupling matrix to the extended box form. In [1] a method was described which is essentially the reverse of the general sequence that reduces any coupling matrix to the folded form, for which a regular sequence of rotation pivots and angles does exist. Using this method means that some of the rotation angles cannot be determined by calculation from the pre-transform coupling matrix (as can be done from the 'forward' method) and so they have to be determined by optimization. Other methods (eg. [2], [3]) are also known to produce a solution. 
Although most target coupling matrix configurations (eg propagating in-line) have one or two unique solutions, the extended box configuration is distinct in having multiple solutions, all returning exactly the same performance characteristics under analysis as the original prototype folded or arrow configuration. The solutions converged upon by existing optimization methods tend to be dependent upon the starting values given to the coupling values or rotation angles, and it can never be guaranteed that all possible solutions have been found. In [4] an approach based on computer algebra was outlined that allows to compute all the solutions for a given coupling matrix topology, including those with complex values (which of course are discarded from the solutions considered for the realization of the hardware). In this paper we detail the latter procedure as well as a modification in the choice of the set of algebraic equations to solve that leads to an important improvement of the algorithm's efficiency in practice.

Having a range of solutions enables a choice to be made of the coupling value set most suited to the technology it is intended to realize the filter with. Considerations influencing the choice include ease of the design of the coupling elements, minimization of parasitic couplings or resonator frequency offsets. Some of the coupling matrix solutions may contain coupling elements with values small enough to be ignored without damage to the overall electrical performance of the filter, so simplifying the manufacture and tuning processes.

In the following section a general proof will be given for the inversion of the frequency characteristics of a network. This is followed by a description of the multi-solution synthesis method, applicable to the extended box network and others that support multiple solutions. Finally we apply our procedure to the synthesis of two filtering characteristics of degree 8 and 10 with respectively 3 and 4 transmission zeros. In each case we demonstrate how the ability to choose among several coupling matrices simplifies the practical realization of the filter in dual-mode waveguide or dielectric resonator cavities. In the $10^{\text {th }}$ degree case, the complete list of equivalent coupling matrices is used to identify couplings corresponding to an iris that can be safely neglected. Finally an approximate synthesis technique based on a postprocessing optimization step is presented and improves the approach of reference [4].

\section{REVERSED FREQUENCY CHARACTERISTICS}

In this section we detail the approach given in [4] concerning the reversion of the response of a filter by means of the sign inversion of some elements of its coupling matrix.

If $S(j w)$ and $G(j w)$ are filter responses we call $\mathrm{G}$ reverted with respect to $\mathrm{S}$ if the following relation holds:

$$
\left|G_{i, j}(j w)\right|=\left|S_{i, j}(-j w)\right| .
$$

The so-called symmetric responses are self-reverted in the latter sense. One striking fact is that the coupling matrices of all usual symmetric filters have a particular geometry, namely, all their couplings $M_{i, j}$ where $(i+j)$ is even are zero. We will say that their coupling matrix is "odd"; a typical example is presented on Fig.2 with a coupling matrix of a 5-2 symmetric filtering characteristic.

\begin{tabular}{|ccccc|}
\hline 0 & 0.11 & 0 & 0 & 0 \\
0.11 & 0 & -0.31 & & 0.12 \\
0 & -0.31 & 0 & 0.21 & 0. \\
0 & 0 & 0.21 & 0 & 0.61 \\
0 & 0.12 & 0 & 0.61 & 0 \\
\hline
\end{tabular}

Fig.2: "Odd" coupling matrix, adapted to a 5-2 symmetric filtering function.

Conversely the coupling matrices of all asymmetric filters have some of their "even" entries $M_{i, j}(i+j$ even) that are nonzero. It is therefore natural to ask about the effects of the inversion of the latter entries. Below it is shown that these sign changes in the coupling matrix are related to the reversion of the filter response.

We say that a matrix $\mathrm{M}$ is "odd" (as opposed to "even" ) if the following holds: for all indices $(i, j)$ such that $(i+j)$ is even (as opposed to "odd") we have $\mathrm{M}[i, j]=0$. It is straightforward that every matrix $\mathrm{M}$ decomposes uniquely in the sum of its odd part (denoted $\mathrm{M}_{\mathrm{o}}$ ) and even part $\left(\mathrm{M}_{\mathrm{e}}\right)$. Now if $\mathrm{M}$ is the $(N \mathrm{x} N)$ coupling matrix of a lossless filter we denote by $y_{i, j}[\mathrm{M}]$ and $S_{i j}[\mathrm{M}]$ the corresponding reduced admittance and scattering parameters (the input and output loads are fixed). The following properties relate in a simple manner the inversion of the even entries of a coupling matrix with the reversion of the associated frequency response.

- $y_{11}\left[\mathrm{M}_{\mathrm{o}}-\mathrm{M}_{\mathrm{e}}\right](s)=-y_{11}\left[\mathrm{M}_{\mathrm{o}}+\mathrm{M}_{\mathrm{e}}\right](-s)$ and the same is true for $y_{22}$

- $y_{12}\left[\mathrm{M}_{\mathrm{o}}-\mathrm{M}_{\mathrm{e}}\right](s)=(-1)^{N} y_{12}\left[\mathrm{M}_{\mathrm{o}}+\mathrm{M}_{\mathrm{e}}\right](-s)$

On the imaginary axis $s=j \omega$,

- $S_{11}\left[\mathrm{M}_{\mathrm{o}}-\mathrm{M}_{\mathrm{e}}\right](j \omega)=\left(S_{11}\left[\mathrm{M}_{\mathrm{o}}+\mathrm{M}_{\mathrm{e}}\right](-j \omega)\right)^{*}$ and the same is true for $S_{22}$.

- $S_{12}\left[\mathrm{M}_{\mathrm{o}}-\mathrm{M}_{\mathrm{e}}\right](j \omega)=(-1)^{N+1}\left(\mathrm{~S}_{12}\left[\mathrm{M}_{\mathrm{o}}+\mathrm{M}_{\mathrm{e}}\right](-j \omega)\right)^{*}$

Proof: From the fact that the product of two square matrices with the same parity is "even" and the product of two square matrices with different parities is "odd" one proves by induction on $k$ that,

$$
\begin{aligned}
& \operatorname{Odd}\left(\left(\mathrm{M}_{\mathrm{o}}-\mathrm{M}_{\mathrm{e}}\right)^{k}\right)=(-1)^{k+1} \operatorname{Odd}\left(\left(\mathrm{M}_{\mathrm{o}}+\mathrm{M}_{\mathrm{e}}\right)^{k}\right) \\
& \operatorname{Even}\left(\left(\mathrm{M}_{\mathrm{o}}-\mathrm{M}_{\mathrm{e}}\right)^{k}\right)=(-1)^{k} \operatorname{Even}\left(\left(\mathrm{M}_{\mathrm{o}}+\mathrm{M}_{\mathrm{e}}\right)^{k}\right)
\end{aligned}
$$

where Odd( ) and Even( ) means respectively taking the odd and the even parts. Now recalling that:

$$
\mathrm{Y}(s)=C(s I-j M)^{-1} C^{t}=\sum_{k=0}^{\infty} \frac{C j^{k} M^{k} C^{t}}{s^{k+1}}
$$


with:

$$
C=\left[\begin{array}{ccc}
\sqrt{R_{1}} & \ldots 0 \ldots & 0 \\
0 & \ldots 0 \ldots & \sqrt{R_{N}}
\end{array}\right]
$$

( $R_{1}, R_{N}$ are the input/output termination impedances), and plugging in the relations (1-2) yields directly the formulae for $\mathrm{Y}$. Finally the classical formula $S=(\mathrm{I}-\mathrm{Y}) /(\mathrm{I}+\mathrm{Y})$ and the fact that $\mathrm{Y}$ is purely imaginary on the imaginary axis leads to the formulae for $S$. In particular this implies that every response associated to an odd coupling matrix is symmetric, i.e. self-reverted.

For asymmetric filters the latter properties mean that to reverse the frequency characteristic of any coupling matrix, elements with indices $(i, j)$ where $(i+j)=$ even are changed in sign, whilst those where $(i+j)=$ odd retain their original sign. Thus for a ladder network such as the extended box network, the elements on the principal diagonal, each of whose indices add to an even integer, need to be changed in sign (ie. conjugate-tuned), to laterally invert the network's response with frequency. All off-diagonal elements retain their original sign, except for $4^{\text {th }}, 8^{\text {th }}, 12^{\text {th }} \ldots$ degree cases where the indices of the last two couplings (eg $M_{68}$ and $M_{57}$ in the $8^{\text {th }}$ degree case, see Fig. 1c) have an index sum that is even. However, in the latter cases applying in addition the diagonal similarity transform that reverts the sign of the last two vectors ( $7^{\text {th }}$ and $8^{\text {th }}$ in the 8 degree case) to the coupling matrix restores the original signs of these couplings without affecting the reverted response of the filter.

\section{A GENERAL FRAMEWORK FOR THE COUPLING MATRIX SYNTHESIS PROBLEM}

In this section we work with a fixed coupling topology, that is we are given a set of independent non-zero couplings associated to a low pass prototype of some filter with $N$ resonators. Starting with numerical values for the couplings and the i/o loads one can easily compute the admittance matrix using equation (3). The coupling matrix synthesis problem is actually about inverting the latter procedure: given an admittance matrix we want to find values for the input/output loads and couplings that realize it. In order to formalize this we give a name to the mapping that builds the admittance matrix from the free electrical parameters and we define:

$$
\begin{aligned}
\mathrm{T}: \mathrm{p}= & \left(\sqrt{R_{1}}, \sqrt{R_{N}} . . M_{i, j}\right) \rightarrow \\
& \left(C C^{t}, \ldots C M^{k} C^{t}, \ldots C M^{2 N-1} C^{t}\right)
\end{aligned}
$$

The above definition is justified by the fact that the admittance matrix is entirely determined by the first $2 \mathrm{~N}$ coefficients of its power expansion at infinity [5].

Now suppose that each of the electrical parameters move around in the complex plane: what about the corresponding set of admittance matrices? The latter can be identified with the image by $\mathrm{T}$ of $\mathrm{C}^{r}$ ( $\mathrm{C}$ is here the field of complex numbers) where $r$ is the number of free electrical parameters. We call this set $\mathrm{V}\left(=\mathrm{T}\left(\mathrm{C}^{r}\right)\right)$ and refer to it as the set of admissible admittance matrices with respect to the coupling topology.

In this setting the coupling matrix synthesis problem is the following: given an element $\mathrm{w}$ in $\mathrm{V}$ compute the solution set of:

$$
\mathrm{T}(\mathrm{p})=\mathrm{w}
$$

Now from the definition of $\mathrm{T}$ it follows that equation (4) is a non-linear polynomial system with $r$ unknowns, namely: the square roots of the i/o loads and the free couplings of the topology. From the polynomial structure of the latter system we can deduce following mathematical properties (we will take them here for granted):

- Equation (4) has a finite number of solutions for all generic $\mathrm{w}$ in $\mathrm{V}$ (generic means for almost all $\mathrm{w}$ in $\mathrm{V}$ ) if and only if the differential of $\mathrm{T}$ is generically of rank $r$. In this case we will say that the coupling topology is non-redundant.

- The number of complex solutions of the equation (4) is generically constant with regard to $w$ in V. Because of the sign symmetries this number is a multiple of $2^{N}$ and can therefore be written as $m 2^{N}$. The number $m$ is the number of complex solutions up to sign symmetries and we will call it the "reduced order" of the coupling geometry.

Remarks:

The non-redundancy property ensures that a coupling geometry is not over-parameterized which would yield a continuum of solutions to our synthesis problem. We illustrate this with the $6^{\text {th }}$ degree topology of Fig. 3 .

- if no diagonal couplings are present (as suggested by the grey dots in Fig. 3), the topology is redundant, ie the synthesis problem admits an infinite number of solutions.

- If, for example, the coupling $(1,4)$ is removed, the topology becomes non-redundant and is adapted to a 6-2 symmetric filtering characteristic. In this case the resulting coupling topology is the so called "arrow form" for which the coupling matrix synthesis problem is known to have only one solution. The reduced order of the latter topology is therefore 1 .

- Finally, if diagonal couplings are allowed, the topology becomes non-redundant, and is actually the $6^{\text {th }}$ degree extended box topology of Fig. 1 and is adapted to a 6-2 asymmetric filtering characteristic. We will see in the following section that its reduced order is 8 .

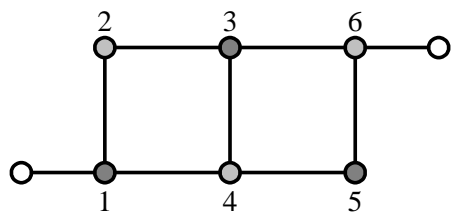

Fig. 3. Redundant topology

The use of the adjective "generic" in the latter statements is necessary for their mathematical correctness. In fact 
properties concerning parameterised algebraic systems are often true for all possible values of the parameters but an exceptional set. An example of this is given by following polynomial:

$$
p(x)=a x^{2}+1
$$

The latter polynomial has two distinct roots for almost all complex values of the parameter $a$ : the exceptional parameter set where the latter property does not hold is characterised by the equation $a=0$ and is very "thin" (or non-generic) as a subset of the complex plan.

The constructive nature of our framework for the synthesis problem depends strongly on our ability to invert numerically the mapping $T$, i.e compute the solution set of equation (4). In the next section we briefly explain how this can be done using Groebner basis computations.

\section{GROEBNER BASIS}

As an example of the use of Groebner basis, suppose we are given the following system:

$$
\left\{\begin{array}{l}
x^{2}+2 x y+1=0 \\
x^{2}+3 x y+y+2=0
\end{array}\right.
$$

By combining equations we get the following polynomial consequences:

$$
\begin{array}{ll}
(b)-(a): & x y+y+1=0 \\
(c) x-(b) y: & 3 x y^{2}-y x-x+y^{2}+2 y=0 \\
(d)-(c) y: & -y x-x-2 y^{2}-y=0 \\
(e)+(c): & -x-2 y^{2}+1=0 \\
(f) y+(c): & -2 y^{3}+2 y+1=0
\end{array}
$$

Note that equation $(g)$ is a univariate polynomial in the unknown $y$. Solving the latter numerically yields the following 3 -digit approximations for $y:\{-0.56+0.25 j,-0.56-0.25 j, 1.19\}$ and from $(f)$ we get the corresponding values for $x=\{0.42-$ $0.61 j, 0.42+0.61 j,-1.84\}$. Now we can verify that the latter three pairs of values for $(x, y)$ are also solutions of $(a)$ and $(b)$ and therefore the only three solutions of our original system. Equations $(f)$ and $(g)$ are what is called a Groebner basis [6] of our original system and allows us to reduce the resolution of a multivariate polynomial system to the one of a polynomial in a single unknown.

The technique that we have presented is a simple example is called "elimination" and can be thought as the non-linear version of the classical Gaussian elimination technique for linear systems. The fact that the process of variables elimination by means of combinations of equations always ends up with a polynomial in a single variable is equivalent to the property that the original system has only isolated solutions [7]. In the case of our synthesis problem this is ensured by the non-redundancy of the considered coupling topology.
In practice, computing a Groebner basis can be computationally very costly: the number of necessary combinations of equations can be very large and strongly grows with the total number of variables of the system. Therefore the use of specialized algorithms and their effective software implementation is strongly recommended. In this work we have used the tool Fgb [8].

Table I summarizes the reduced order and the number of real solutions observed for a particular filtering characteristic for each of the extended box networks of Fig.1. The synthesis method is not limited to the case of extended box topologies: Table I also mentions the case of a $10^{\text {th }}$ degree topology (Fig. 4) adapted to $10-8$ symmetric characteristics. The reduced order of the latter is equal to 3 and is therefore much smaller than the reduced order of 384 of its $10^{\text {th }}$ degree extended box analogue. This is something we observed empirically by testing our method on various networks: topologies adapted to asymmetric characteristics seem to have a much higher reduced order than those adapted to symmetric ones.

Whereas the reduced order depends only on the coupling geometry, the number of real solutions depends on the prototype characteristic the network is realizing (position of transmission zeros (TZs), return loss, etc...) and is, by definition, bounded from above by the reduced order. One can even construct some coupling topologies and some filtering characteristics for which the synthesis problem admits only complex solutions. An academic example of this is given by the topology of Fig. 5 and the filtering characteristic, the canonical coupling matrix in arrow form of which is given on Fig. 6. In this latter case the reduced order of the coupling topology is 2 but both solutions to the synthesis problem are complex and equal to the matrix of Fig. 7 and to its conjugate.

TABLE I

REDUCED ORDER \& OBSERVED NUMBER OF REAL SOLUTIONS

\begin{tabular}{c|c|c|c}
\hline Topology & $\begin{array}{c}\text { Max. No. of } \\
\text { TZs }\end{array}$ & $\begin{array}{c}\text { Reduced } \\
\text { Order }\end{array}$ & $\begin{array}{c}\text { Observed No. of } \\
\text { Real Solutions }\end{array}$ \\
\hline \hline Fig. 1(a) & 1 & 2 & 2 \\
Fig. 1(b) & 2 & 8 & 6 \\
Fig. 1(c) & 3 & 48 & 16 \\
Fig. 1(d) & 4 & 384 & 36,58 \\
Fig. 4 & 8 & 3 & 1 \\
\hline \hline
\end{tabular}

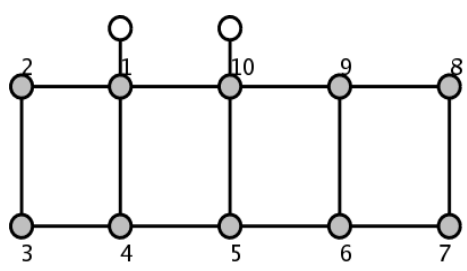

Fig. 4. Coupling topology adapted to $10-8$ symmetric characteristics. 


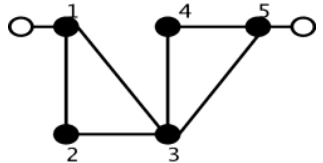

Fig. 5. Academic example of a $5^{\text {th }}$ degree coupling topology adapted to 5-2 asymmetric characteristics.

\begin{tabular}{|lllll|}
\hline 0 & 0.4 & 0 & 0 & 0 \\
0.4 & 0.3 & 0.1 & 0 & 0.1 \\
0 & 0.1 & .2 & 0.2 & 0.2 \\
0 & 0 & 0.2 & 0.2 & 1 \\
0 & 0.1 & 0.2 & 1 & 0.1 \\
\hline
\end{tabular}

Fig. 6. Canonical coupling matrix in "arrow form" of a 5-2 filtering function, admitting only complex coupling matrices when using the topology of Fig. 5.

\begin{tabular}{|lllll|}
\hline 0 & $0.41-0.001 \mathrm{j}$ & $0.006+0.074 \mathrm{j}$ & 0 & 0 \\
$0.41-0.001 \mathrm{j}$ & $0.3-0.035 \mathrm{j}$ & $0.079+0.031 \mathrm{j}$ & 0 & 0 \\
$0.006+0.074 \mathrm{j}$ & $0.079+0.031 \mathrm{j}$ & $.099-0.2 \mathrm{j}$ & $0.3-0.075 \mathrm{j}$ & $0.043-0.54 \mathrm{j}$ \\
0 & 0 & $0.3-0.075 \mathrm{j}$ & $0.3+0.23 \mathrm{j}$ & $1.2+0.02 \mathrm{j}$ \\
0 & 0 & $0.043-0.54 \mathrm{j}$ & $1.2+0.02 \mathrm{j}$ & 0.1 \\
\hline
\end{tabular}

Fig. 7. Complex solution to the synthesis problem with coupling topology of Fig. 5 and coupling matrix in canonical arrow form of Fig. 6.

\section{PRACTICAL IMPLEMENTATION OF THE SYNTHESIS PROCEDURE AND EXAMPLES}

\section{A. $8^{\text {th }}$ Degree Extended Box Filter.}

As an application we will consider the synthesis of an $8^{\text {th }}$ degree filter in extended box configuration (see Fig. 1c). Using a computer algebra system (eg. Maple) we check that that this topology is non-redundant and from the application of the minimum path rule we conclude that the set of admissible admittances consists of rational reciprocal matrices of degree 8 with at most 3 transmission zeros. Using classical quasielliptic synthesis techniques an eighth degree filtering characteristic is designed with a $23 \mathrm{~dB}$ return loss and three prescribed TZs producing one rejection lobe level of $40 \mathrm{~dB}$ on the lower side and two at 40dB on the upper side (see Fig. 8a).

(a)

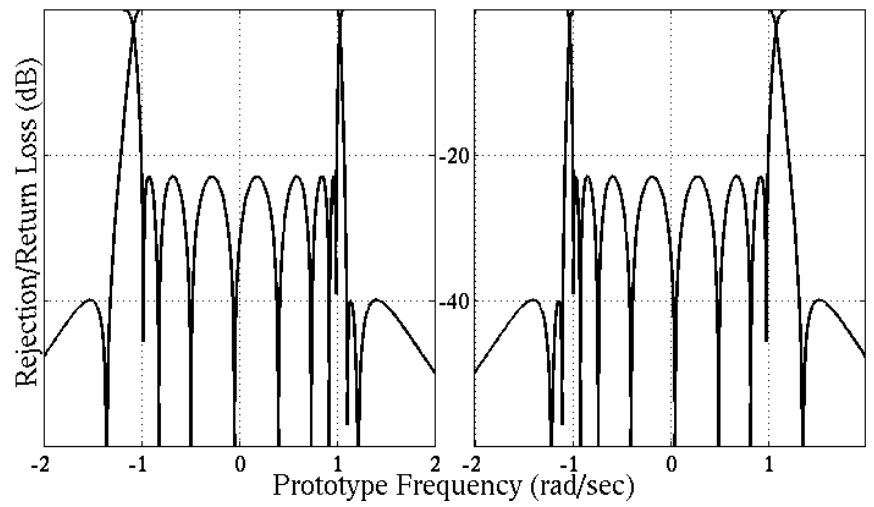

Fig. 8. (a) Original and (b) inverted rejection and return loss performance of an 8-3 asymmetric characteristic in extended box configuration.

Now computing the $2 \mathrm{~N}$ first terms of the power expansion of the admittance matrix yields the left hand term of (4) which in turn could be solved using Groebner basis computations. At this point it is important to mention that the complexity of the Groebner basis computations of a system increases with its total number of complex solutions. The natural sign symmetries of the system derived from equation (4) tend to artificially increase the latter (total number of solutions $=\mathrm{m} 2^{\mathrm{N}}$ ) and may dramatically increase the computation time of the corresponding Groebner basis. Before continuing on with the synthesis we therefore explain how a rewriting of equation (4) allows us to get rid of these unwanted sign symmetries.

An alternative to equation (4) to invert the mapping $T$ is to use an algebraic version of the approach presented in [9] that is based on similarity transforms. If $M$ is a coupling matrix in canonical form realizing the admittance matrix then equation (4) is "equivalent" to the following matrix equation where the unknown is a similarity transform $P$.

$$
\begin{aligned}
& P=\left(\begin{array}{ccc}
1 & \ldots 0 \ldots & 0 \\
\vdots & H & \vdots \\
0 & \ldots 0 \ldots & 1
\end{array}\right) \\
& H^{t} H=I d \\
& \forall(i, j) \in I \quad\left(P^{t} M P\right)_{i . j}=0
\end{aligned}
$$

In the latter, $I$ is the set of indices corresponding to the couplings that must be zero in the target topology (in our example $I=\{(1,3),(1,5),(1,6) \ldots \ldots\})$. If $P$ is a solution of $(5)$ it is readily seen that all the similarity transforms that are obtained from $P$ by inverting some of the columns vectors of the submatrix $H$ are also solutions of (5). In order to break these symmetries the "trick" is to slightly modify (5b). We denote by $h_{i}$ the $\mathrm{i}^{\text {th }}$ column vector of $H$. Some of the equations of (5b) indicate that the vectors $h_{i}$ are unitary with regard to the Euclidean norm. We replace these normalizing equations by:

$$
u_{i}^{t} h_{i}=1
$$

where $u_{i}$ is a randomly-chosen vector. We call (5') the resulting system. It can be verified that for a generic choice of the $u_{i}$ 's, all the solutions of (5) that are equivalent up to sign changes of their column vectors correspond to a single solution of (5'). More precisely to every set of solutions of (5) of the form:

$$
H=\left( \pm h_{1}, \pm h_{2} \ldots \pm h_{i} \ldots\right)
$$

there corresponds a unique solution $\mathrm{G}=\left(\mathrm{g}_{1} \ldots \mathrm{g}_{\mathrm{i}} \ldots\right)$ of $\left(5^{\prime}\right)$ where the column vectors $g_{i}$ are given by: 


$$
g_{i}=\frac{h_{i}}{u_{i}^{t} h_{i}}
$$

With regard to the Groebner basis computation system, (5') has shown to be much more tractable than the algebraic system derived from equation (4).

Getting back to our $8^{\text {th }}$ degree example, we compute $M$ the associated coupling matrix in arrow form and set up (5'). The latter is an algebraic system of linear and quadratic equations in the entries of $H$. The computation of its Groebner basis leads to the following result:

- the reduced order of the topology is 48

- for this particular filtering characteristic, 16 of the 48 solutions are real-valued.

Only the real solutions have a physical interpretation and are therefore of practical interest.

The criterion used to choose the best coupling matrix out of the 16 realizable ones will depend on the hardware implementation of the filter. Having in mind a realization with dual mode cavities, we choose to select solutions where the asymmetry between the two "arms" of each cross-iris is maximized in order to minimize parasitic couplings. The best ratios between couplings of the relevant pairs $\left(M_{14}, M_{23}\right),\left(M_{36}\right.$, $\left.M_{45}\right)$ and $\left(M_{57}, M_{68}\right)$ are found for the solution shown in Fig.9a, where each cross-iris has one of its coupling values at least 5 times larger than the other one.

\begin{tabular}{|cccccccc|}
\hline 0.0107 & -0.2904 & 0 & -0.8119 & 0 & 0 & 0 & 0 \\
-0.2904 & -0.9804 & 0.1081 & 0 & 0 & 0 & 0 & 0 \\
0 & 0.1081 & 0.0605 & 0.5475 & 0 & 0.5984 & 0 & 0 \\
0.8119 & 0 & 0.5475 & 0.1384 & -0.0663 & 0 & 0 & 0 \\
0 & 0 & 0 & -0.0663 & 0.0152 & 0.5334 & 0.6782 & 0 \\
0 & 0 & 0.5984 & 0 & 0.5334 & 0.0226 & 0 & -0.1260 \\
0 & 0 & 0 & 0 & 0.6782 & 0 & 0.0113 & 0.8530 \\
0 & 0 & 0 & 0 & 0 & -0.1260 & 0.8530 & 0.0107 \\
\hline
\end{tabular}

(a)

\begin{tabular}{|cccccccc|}
\hline 0.0107 & 0.0001 & 0 & -0.2464 & 0 & 0 & 0 & 0 \\
0.0001 & -0.9590 & 0.2094 & 0 & 0 & 0 & 0 & 0 \\
0 & 0.2094 & 0.0498 & 0.4681 & 0 & -0.4681 & 0 & 0 \\
-0.2464 & 0 & 0.4681 & 0.0115 & 0.3744 & 0 & 0 & 0 \\
0 & 0 & 0 & 0.3744 & -0.0439 & 0.3744 & 0.8165 & 0 \\
0 & 0 & -0.4681 & 0 & 0.3744 & 0.0115 & 0 & 0.8623 \\
0 & 0 & 0 & 0 & 0.8165 & 0 & 0.1975 & 0.0001 \\
0 & 0 & 0 & 0 & 0 & 0.8623 & 0.0001 & 0.0107 \\
\hline
\end{tabular}

(b)

Fig. 9. ' $N \mathrm{x} N$ ' coupling matrices for an 8-3 asymmetric prototype: a) extended box configuration, b) 'cul-de-sac' configuration. $R_{1}=$ $R_{N}=1.0878$

Fig. 9b illustrates that sometimes solutions emerge which have very small values for certain couplings $\left(M_{12}\right.$ and $M_{78}$ in this case), which may be safely omitted for the implementation without damaging the final response of the network. In this case a quasi cul-de-sac network is produced, similar to the 8-3 example given in [1]. In fact one can show that with some renumbering, the cul-de-sac network of [1] is a sub-topology of the extended box where the couplings $M_{12}$ and $M_{78}$ are set to zero. The cul-de-sac topology is more restrictive than the extended box one in the sense that it is only adapted for the synthesis of "double-terminated" characteristics. However our current filtering characteristic is, up to numerical errors, "double-terminated" and this explains why in this example a quasi cul-de-sac network is found among all possible coupling matrices.

Finally, using the result of Section II, it is shown that only the resonators need to be retuned in order to obtain an inverted characteristic. Fig. 8(b) shows the rejection and return loss obtained from the coupling matrices of Fig. 9 when the signs of their diagonal elements $M_{i, i}$ are changed.

\section{B. $10^{\text {th }}$ Degree Extended Box Filter and approximate synthesis technique}

We consider the synthesis of a $10^{\text {th }}$ degree filter in the extended box topology of Fig. 1(d). Using our procedure we check that this topology is non-redundant and that it is adapted to asymmetric characteristics with up to $4 \mathrm{TZs}$. A filtering characteristics is designed with a $23 \mathrm{~dB}$ return loss, $2 \mathrm{TZs}$ at $+j 1.10929$ and $+j 1.19518$ to give two $50 \mathrm{~dB}$ rejection lobes on the upper side, and 2 more complex zeros at $\pm 0.75877-j 0.13761$ for group delay equalization purposes (see Fig. 11).

The corresponding coupling matrix in arrow form is determined and the computation of a Groebner basis of system (5') yields the following:

- The reduced order of the topology is 384

- For our specific filtering characteristic 36 real and therefore realizable solutions are found

When realized with dual mode cavities this topology requires 4 cross- irises. Our aim is to demonstrate how our exhaustive approach may allow the "replacement" of a crossiris by an iris with a single arm as well as to simplify the future computer-aided tuning process of the filter.

Amongst all the possible coupling matrices the one with the smallest coupling corresponding to an iris is selected, which leads to the matrix of Fig. 12 where $M_{45}$ is equal to -0.001 . Setting $M_{45}$ to zero yields a small but undesirable variation of the return loss as well as of the upper-band rejection lobes. The remaining couplings are therefore re-tuned thanks to an optimization step that minimizes the discrepancy between the original response and the one obtained by imposing that $M_{45}$ be zero (see Fig. 13 for the resulting coupling matrix). A quasi perfect fit is obtained between the two responses: the least square error between the two return losses on the normalized broadband $[-3,3]$ equals $8.83 .10^{-5}$ (on the Bode plot there is visually no difference).

Finally the simplified coupling topology of Fig. 10 is considered as a new topology in its own right. Using our procedure its reduced order is found to be equal to 2 and a second equivalent coupling matrix with the same coupling 
topology is computed (see Fig. 14). With regard to the "iris asymmetry criterion" of the last section the latter matrix is the best one.

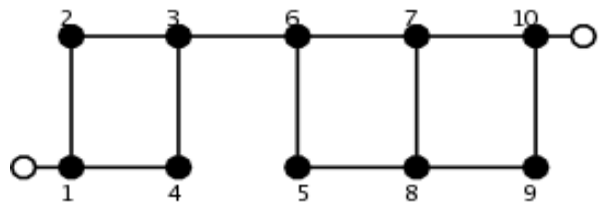

Fig. 10. Simplified $10^{\text {th }}$ degree topology.

Note that besides the removal of a cross-iris we have also lowered the reduced order of our target topology from 384 to 2. This is important if one wants to use a computer aided tuning process [10] that typically identifies a coupling matrix from measured data. In the cases of topologies with multiple solutions, such a tool will return a set of equivalent coupling matrices and leave to the user the 'expert' task of choosing the "right" one. This can be done by using some extra information concerning the physical device, like for example an a priori estimation of the coupling value realizable by some irises. Nevertheless the latter task is of course much easier to carry out with a short list of equivalent coupling matrices than with a huge one.

\section{CONCLUSION}

In this paper, a new method for the synthesis of the full range of coupling matrices for networks that support multiple solutions is presented. This procedure yields an exhaustive list of all the solutions to the synthesis problem. Based on the latter, an approximate synthesis technique is derived which allows the reduction of the constructional complexity of highdegree asymmetric filters in dual-mode technologies. In addition it has been shown that a knowledge of which solutions are possible is important when reconstructing the coupling matrix from measured data, during development or computer-aided tuning (CAT) processes. Also the property of reversibility of frequency characteristics by tuning alone has been proved.

\section{REFERENCES}

[1] R. J. Cameron, A. R. Harish and C. J. Radcliffe, "Synthesis of advanced microwave filters without diagonal cross-couplings," IEEE Trans. Microwave Theory Tech., vol. MTT-50, pp. 2862-2872, Dec. 2002.

[2] G. Macchiarella, "A powerful tool for the synthesis of prototype filters with arbitrary topology," 2003 IEEE MTT-S Int. Microwave Symp. Dig., vol. 3, pp. 1721-1724, June 2003.

[3] S. Amari, "Synthesis of Cross-Coupled Resonator Filters Using an Analytical Gradient-Based Optimization Technique", IEEE Trans. Microwave Theory Tech., vol. MTT-48, pp. 1559-1564, Sept. 2000.

[4] R.J. Cameron, J.C. Faugere, F. Seyfert, "Coupling Matrix Synthesis for a New Class of Microwave Filter Configuration", 2005 IEEE MTT-S International Microwave Symp. Dig., June 2005.

[5] T. Kailath, "Linear Systems", Prentice Hall, 1980.

[6] D. Cox, J. Little and D. O'Shea, "Ideals, Varieties, and Algorithms", Springer, Berlin, 1997.

[7] L. Gonzalez-Vega, F. Rouillier and M.F Roy, "Some tapas of computer algebra", Algorithms and Computation in Mathematics, vol. 4, Springer, Berlin, 1999.

[8] J. C. Faugere, "A new efficient algorithm for computing Groebner bases without reduction to zero (F5)", Proc. Int. Symp. on Symbolic and Algebraic Comp., pp. 75-83, New York, May 2002.

[9] R.J. Cameron, "General Coupling Matrix Synthesis Method for Chebyshev Filtering Functions", IEEE Trans. Microwave Theory Tech., vol. MTT-47, pp. 433-442, April1999.

[10]F. Seyfert, L. Baratchart, J.P. Marmorat, S. Bila, J. Sombrin, "Extraction of Coupling Parameters For Microwave Filters: Determination of a Stable Rational Model from Scattering Data”, 2003 IEEE MTT-S International Microwave Symp. Dig., vol 1, pp. 25-28, June 2003. 


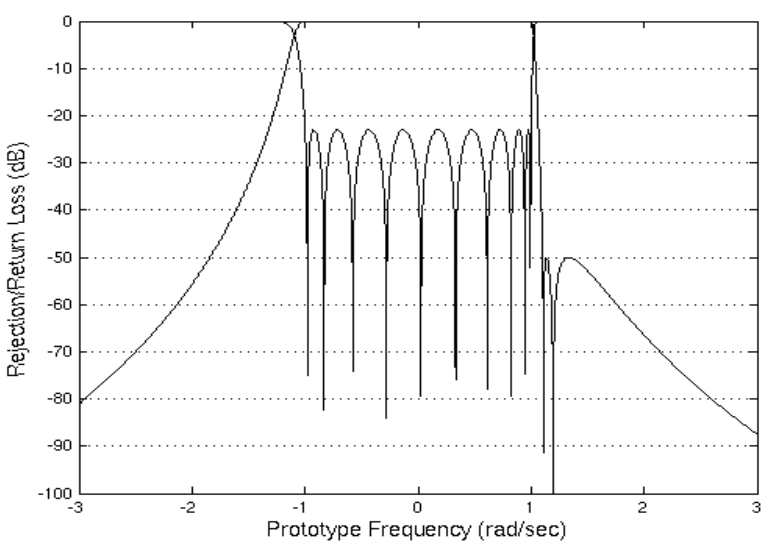

(a)

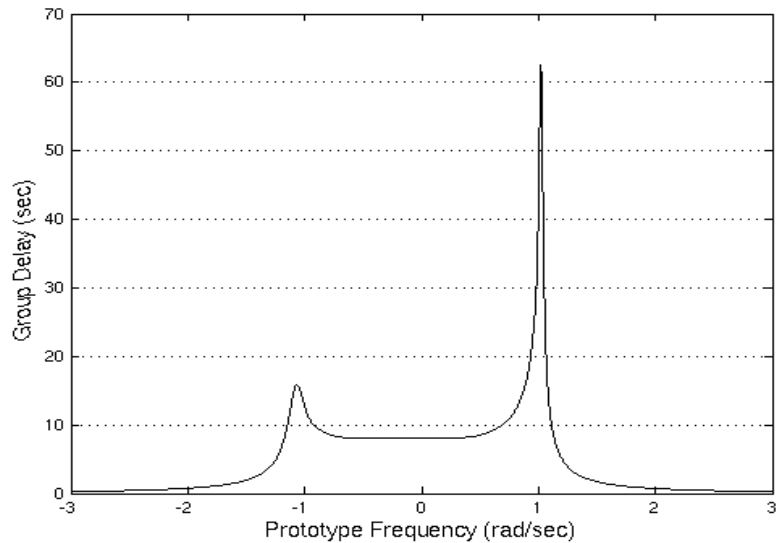

(b)

Fig. 11. 10-2-2 asymmetric characteristic: (a) rejection and return loss (b) group delay.

\begin{tabular}{|lllllllll|}
\hline 0.0145 & 0.7712 & 0 & 0.3879 & 0 & 0 & 0 & 0 & 0 \\
0.7712 & 0.2493 & -0.5232 & 0 & 0 & 0 & 0 & 0 & 0 \\
0 & -0.5232 & 0.0554 & 0.1925 & 0 & -0.5393 & 0 & 0 & 0 \\
0.3879 & 0 & 0.1925 & -0.9071 & -0.0010 & 0 & 0 & 0 & 0 \\
0 & 0 & 0 & -0.0010 & -0.7492 & -0.2683 & 0 & 0.3110 & 0 \\
0 & 0 & -0.5393 & 0 & -0.2683 & 0.0437 & -0.4668 & 0 & 0 \\
0 & 0 & 0 & 0 & 0 & -0.4668 & 0.3195 & -0.4934 & 0 \\
0 & 0 & 0 & 0 & 0.3110 & 0 & -0.4934 & -0.1000 & 0.4827 \\
0 & 0 & 0 & 0 & 0 & 0 & 0 & 0.4827 & -0.0021 \\
0 & 0 & 0 & 0 & 0 & 0 & -0.2040 & 0 & 0.8388 \\
\hline
\end{tabular}

Fig. 12. Coupling matrix of the 10-2-2 characteristic of Fig. 11 with the extended box topology and a "small" $M_{45}$ coupling, $R_{1}=R_{N}=1.04326$.

\begin{tabular}{|llllllllll|}
\hline 0.0161 & 0.7655 & 0 & 0.4053 & 0 & 0 & 0 & 0 & 0 \\
0.7655 & 0.2705 & -0.5173 & 0 & 0 & 0 & 0 & 0 & 0 \\
0 & -0.5173 & 0.0560 & 0.2057 & 0 & -0.5386 & 0 & 0 & 0 \\
0.4053 & 0 & 0.2057 & -0.8923 & 0 & 0 & 0 & 0 & 0 \\
0 & 0 & 0 & 0 & -0.7810 & -0.2512 & 0 & 0.2968 & 0 & 0 \\
0 & 0 & -0.5386 & 0 & -0.2512 & 0.0445 & -0.4761 & 0 & 0 \\
0 & 0 & 0 & 0 & 0 & -0.4761 & 0.2867 & -0.5041 & 0 \\
0 & 0 & 0 & 0 & 0.2968 & 0 & -0.5041 & -0.0850 & 0.4851 \\
0 & 0 & 0 & 0 & 0 & 0 & 0 & 0 & 0.4851 & 0.0016 \\
0 & 0 & 0 & 0 & 0 & 0 & -0.1984 & 0 & 0.8427 \\
\hline
\end{tabular}

Fig. 13. Coupling matrix of the 10-2-2 characteristic of Fig. 11 with a simplified topology, (i.e. $\left.M_{45}=0\right), R_{1}=1.0969, R_{N}=1.0963$.

\begin{tabular}{|lllllllll|}
\hline 0.0161 & 0.4053 & 0 & 0.7655 & 0 & 0 & 0 & 0 & 0 \\
0.4053 & -0.8923 & -0.2057 & 0 & 0 & 0 & 0 & 0 & 0 \\
0 & -0.2057 & 0.0560 & 0.5173 & 0 & -0.5386 & 0 & 0 & 0 \\
0.7655 & 0 & 0.5173 & 0.2705 & 0 & 0 & 0 & 0 & 0 \\
0 & 0 & 0 & 0 & -0.7810 & -0.2512 & 0 & 0.2968 & 0 \\
0 & 0 & -0.5386 & 0 & -0.2512 & 0.0445 & -0.4761 & 0 & 0 \\
0 & 0 & 0 & 0 & 0 & -0.4761 & 0.2867 & -0.5041 & 0 \\
0 & 0 & 0 & 0 & 0.2968 & 0 & -0.5041 & -0.0850 & -0.4851 \\
0 & 0 & 0 & 0 & 0 & 0 & 0 & -0.4851 & 0.0016 \\
0 & 0 & 0 & 0 & 0 & 0 & 0.1984 & 0 & 0.8427 \\
\hline
\end{tabular}

Fig. 14. Coupling matrix with a simplified topology and the most asymmetric irises, $R_{1}=1.0969, R_{N}=1.0963$. 